\title{
Testing the recent theories for the origin of the hermaphrodite flower by comparison of the transcriptomes of gymnosperms and angiosperms
}

\author{
Raquel Tavares ${ }^{1 *}$, Mathilde Cagnon ${ }^{1}$, Ioan Negrutiu², Dominque Mouchiroud ${ }^{1}$
}

\begin{abstract}
Background: Different theories for the origin of the angiosperm hermaphrodite flower make different predictions concerning the overlap between the genes expressed in the male and female cones of gymnosperms and the genes expressed in the hermaphrodite flower of angiosperms. The Mostly Male (MM) theory predicts that, of genes expressed primarily in male versus female gymnosperm cones, an excess of male orthologs will be expressed in flowers, excluding ovules, while Out Of Male (OOM) and Out Of Female (OOF) theories predict no such excess.

Results: In this paper, we tested these predictions by comparing the transcriptomes of three gymnosperms (Ginkgo biloba, Welwitschia mirabilis and Zamia fisheri) and two angiosperms (Arabidopsis thaliana and Oryza sativa), using EST data. We found that the proportion of orthologous genes expressed in the reproductive organs of the gymnosperms and in the angiosperms flower is significantly higher than the proportion of orthologous genes expressed in the reproductive organs of the gymnosperms and in the angiosperms vegetative tissues, which shows that the approach is correct. However, we detected no significant differences between the proportion of gymnosperm orthologous genes expressed in the male cone and in the angiosperms flower and the proportion of gymnosperm orthologous genes expressed in the female cone and in the angiosperms flower.

Conclusions: These results do not support the MM theory prediction of an excess of male gymnosperm genes expressed in the hermaphrodite flower of the angiosperms and seem to support the OOM/OOF theories. However, other explanations can be given for the 1:1 ratio that we found. More abundant and more specific (namely carpel and ovule) expression data should be produced in order to further test these theories.
\end{abstract}

\section{Background}

In spite of the great and ever growing amount of morphological and molecular data accumulating from paleobotany, phylogenetics and evo-devo analysis, the origin of the angiosperms hermaphrodite flower is still the "abominable mistery" Charles Darwin referred to, in a letter written to the British botanist Joseph Dalton Hooker, 150 years ago [1-3].

Many theories have been proposed to explain angiosperm origins, differing on the features proposed for the

\footnotetext{
* Correspondence: raquel.tavares@univ-lyon1.fr

'Université de Lyon, F-69622, Lyon, France; Université de Lyon 1, Villeurbanne; CNRS, UMR5558, Laboratoire Biométrie et Biologie Évolutive Villeurbanne, France

Full list of author information is available at the end of the article
}

ancestor of the flower and on the evolutionary mechanisms giving rise to the "modern" hermaphrodite structure. The most recent ones have the advantage that they can be tested using molecular data from extant plants, namely gymnosperms and angiosperms [4]. One of these recent hypotheses, the "Mostly Male Theory", suggests that the angiosperms flower derives from the male reproductive structures of the ancestor, on which ectopic ovules (normally located on the female axis) have developed. The male unit would thus become bisexual and later some microsporophylls (modified leaves bearing the male structures producing microspores) would have enclosed the ovules, giving rise to the angiosperms carpel [5]. Alternatively, the Out Of Male and the Out of Female theories propose that homeotic changes in 
gene expression would have given rise to a perianth-less flower-like structure (a flower where the reproductive structures are not protected, for instance by petals or sepals) with male reproductive units in the basal region and female reproductive units in the apical region [6,4].

These theories make different predictions concerning the overlap between the genes expressed in the male and female cones of gymnosperms and the genes expressed in the hermaphrodite flower of angiosperms. Since it states that the ancestral flower derived from a mainly male axis (on which only the ovules would be female) the Mostly Male (MM) Theory predicts an excess of orthologs of gymnosperm male genes expressed in the angiosperms flower. In other words, of the genes expressed in the hermaphrodite flower, "more should have close homologs (or orthologs, if gene trees are sufficiently resolved to demonstrate orthology) active in male gymnosperm reproductive structures rather than in female structures " [5]. On the other hand, proposing a "mixed" structure, half male half female, as the flower ancestor, the Out Of Male (OOM) and the Out Of Female (OOF) theories predict no excess of gymnosperm male (or female) cone genes expressed in the angiosperm flower [7].

The Floral Genome Project [8] has generated abundant sequence collections of several gymnosperm and angiosperm species. Large EST datasets, capturing thousands of sequences of genes expressed during flower development in each species, have been accumulated.

Using both data from the FGP and from the Arabidopsis [9] and rice genome projects $[10,11]$, we tested these predictions by comparing the transcriptomes of three gymnosperms (Ginkgo biloba, Welwitschia mirabilis and Zamia fisheri) and two angiosperms (Arabidopsis thaliana and Oryza sativa). We chose Arabidopsis and rice for the abundance of EST data and because their complete genome is sequenced, which ensures that the "right" angiosperm ortholog is found for each gymnosperm gene. We used all gymnosperm species for which we had enough EST data from male and female cones' libraries. This is unfortunately not the case for Pinus taeda, from which not such libraries exist, despite the total of more than 300000 EST sequences available from this species.

\section{Methods}

\section{Coding sequences data}

cDNA and peptide sequences of the angiosperms were retrieved from the Rice Genome Annotation Project http://rice.plantbiology.msu.edu/ release 5-Jan 2007-66 710 sequences) $[10,11]$ and from The Arabidopsis Information Resource (TAIR) http://www.arabidopsis.org/ TAIR8 release - April 2008-38 963 sequences) [9]. Unigene sequences of the gymnosperms were retrieved from The Floral Genome Project http://fgp.bio.psu.edu/ Welwitschia mirabilis: 6214 unigenes and Zamia fischeri: 9967 unigenes) [8] and the New York Plant Genomics Consortium http://nypgenomics.org/Ginkgo biloba: 3820 unigenes made by Brenner and collaborators [12]).

\section{Expression data}

We used total of 1526133 EST from Arabidopsis thaliana, 1220876 EST from Oryza sativa, 10129 EST from Welwitschia mirabilis, 8252 EST from Zamia fischeri and 6250 EST from Ginkgo biloba (dbEST release 080108 GenBank, NCBI). EST libraries obtained from mRNA extractions of the same organs, types of libraries, developmental stages and physiological conditions (called here expression categories) were grouped, according to the annotations provided by the NCBI (library.report). We generated complete expression data for the 66710 rice cDNA and for the 38963 Arabidopsis cDNA, using all the ESTs from a total of 317 rice libraries (grouped in 51 expression categories) and 181 Arabidopsis libraries (39 expression categories). In order to perform our tests, we then selected the expression data from flowers and inflorescences (angiosperms), male and female (early developmental stages) cones (gymnosperms) and vegetative tissues (leaves and pools of vegetative organs). Table 1 summarizes the information of the EST numbers and libraries used in these comparisons.

Expression data within each species was obtained by linking the EST to the cDNA (or unigenes) of the species. This was made by blastn between the cDNA (unigenes) sequences (query) and the EST sequences (bank). A threshold alignment score of E-10 was used to filter the results, and only the alignments of at least $100 \mathrm{bp}$ and with a minimum of $95 \%$ of identity between the sequences were retained. Multiple hits of the same EST to one cDNA sequence were discarded (only the best was retained) and whenever the same EST aligned with different cDNA, we kept only the hits having a score of at least $90 \%$ of the score of the best hit. One "good" hit (respecting the criteria described above) with an EST was sufficient for a gene to be considered expressed in the organ corresponding to the EST library. No quantitative analysis of the expression level was performed, only the presence/absence of each gene in the different EST libraries was considered.

\section{Orthology}

The orthology between the angiosperm and gymnosperm genes was established by the best reciprocal hit method. This means that a blast is performed between the two species genes using each species both as the query and as the subject for the alignments. The best 
Table 1 Number of ESTs per library used in the reproductive/vegetative and male/female comparisons

\begin{tabular}{|c|c|c|c|c|c|}
\hline \multicolumn{6}{|l|}{ EST data } \\
\hline \multicolumn{2}{|l|}{ Arabidopsis } & \multicolumn{2}{|l|}{ Rice } & & \\
\hline ORGANS & EST nber & ORGANS & EST nber & & \\
\hline flower (4 lib) & 8551 & fl+pan (44 lib) & 256423 & & \\
\hline flower+infl (8 lib) & 9927 & leaf (20 lib) & 105113 & & \\
\hline leaf+rosette (8 lib) & 2913 & veg pool (2 lib) & 30398 & & \\
\hline veg pool (2 lib) & 2501 & & & & \\
\hline TOTAL EST nber & 23892 & TOTAL EST nber & 391934 & & \\
\hline \multicolumn{2}{|l|}{ Ginkgo } & \multicolumn{2}{|l|}{ Welwitschia } & \multicolumn{2}{|l|}{ Zamia } \\
\hline ORGANS & EST nber & ORGANS & EST nber & ORGANS & EST nber \\
\hline $\begin{array}{l}\text { female cone } \\
\text { (1 lib) }\end{array}$ & 2117 & $\begin{array}{l}\text { female strob } \\
\text { (1 lib) }\end{array}$ & 5283 & $\begin{array}{l}\text { megasp+ovu } \\
\text { (1 lib) }\end{array}$ & 3711 \\
\hline male cone (1 lib) & 2047 & microsp (2 lib) & 4846 & microsp (1 lib) & 4541 \\
\hline female leaf (1 lib) & 1061 & & & & \\
\hline male leaf (1 lib) & 1025 & & & & \\
\hline TOTAL EST nber & 6250 & TOTAL EST nber & 10129 & TOTAL EST nber & 8252 \\
\hline
\end{tabular}

nber: number; lib: library(ies); infl: inflorescence; veg: vegetative; fl: flower; pan: panicle; strob: strobili; megasp: megasporophyll; microsp: microsporopyll; ovu: ovule.

hit for each query sequence is retained, the results of the two (reciprocal) blast are compared, and only the pairs of sequences corresponding to a best hit in both blast are retained as pairs of orthologs.

Blast was performed between the peptide sequences mentioned above for Arabidopsis and rice and either peptide (Ginkgo biloba) or nucleotide (all three gymnosperms) sequences of the gymnosperms. The peptide sequences of G.biloba were obtained applying a polypeptide prediction pipeline, prot4EST [13] to the unigene sequences. For this species, orthology with Arabidopsis and rice was established by reciprocal best hit using both blastp between the peptide sequences of the two species or blastx and tblastn between the peptide sequences of Arabidopsis and rice and the nucleotide sequences of G.biloba. As the results were very similar, orthologs between the other two gymnosperms and Arabidopsis and rice were determined by reciprocal best hit using blastx and tblastn between the peptide sequences of Arabidopsis and rice and the nucleotide sequences of the gymnosperms (i.e. no peptide sequence predictions were used for the gymnosperms). All blast results were filtered with an E-10 threshold for the alignment score.

\section{Biological tests}

The test of the gene expression predictions of the theories for the origin of the hermaphrodite flower was done by comparing the number of genes expressed both in the male cone of the gymnosperms and in the angiosperm flower with the number of genes expressed both in the female cone of the gymnosperms and in the angiosperm flower. It is thus a male/female comparison. We did not compare the absolute number of genes, this value has instead been "normalized" by the number of genes expressed in each cone (and thus eliminating the influence of the size of the EST library) or, more precisely, by the number of genes expressed in each cone for which we were able to find an ortholog in the angiosperm species.

A consequence of the relative small size of the gymnosperm EST libraries is that we most certainly do not detect all the genes actually expressed in each tissue. This means that we may not use this type of data to detect tissue specific genes (any false negative in a tissue expression set would produce a false positive in another tissue specific expression set). We thus made the analysis using the total set of genes expressed in each male or female cone of the gymnosperms, independently of its expression in the other, female or male, cone.

In order to evaluate the suitability of the expression EST data for this kind of test, we made other comparisons, which worked as a control of the main analysis. One strong hypothesis that legitimates Frohlich's and Parker's predictions [5] is that differences in the tissue identity (which, in this case, is used as an indicator of the tissue origin) can be evaluated at a transcriptomics level by comparing proportions of expressed genes. If this is true, we should expect, for instance, the proportion of genes expressed in common in the reproductive organs of a gymnosperm and the flower of an angiosperm to be greater than the proportion of genes expressed in common in the reproductive organs of a gymnosperm and the vegetative organs of an angiosperm. We tested this by comparing the proportion of genes having an angiosperm ortholog and being expressed in each gymnosperm male and female cones 
and in a reproductive organ of the angiosperms with the proportion of genes having an angiosperm ortholog and being expressed in each gymnosperm male and female cones and in a vegetative organ of the angiosperms. Two kinds of pools of reproductive organs (flower and flower and inflorescences) and of vegetative organs (leaves and pools of vegetative organs) were used in the analysis. We did this for the three gymnosperms and the two angiosperms, making a total of 36 "control" comparisons.

\section{Statistical analysis}

The statistical evaluation of the expression ratios was made by 1 ) estimating the confidence interval (CI) of the proportion of the gymnosperm genes (with an angiosperm ortholog) expressed in a gymnosperm organ, from which the angiosperm orthologs are expressed in an angiosperm organ. The choice of using a CI estimation of a frequency instead of the frequency itself was made because the total $n$ (number of orthologs found between the two species that are expressed in a gymnosperm organ) is not the same for the different proportions calculated; 2) calculating the ratio between two of these CI (for instance, between the CI of the expression in a gymnosperm male cone and an angiosperm flower versus the $\mathrm{CI}$ of the expression in a gymnosperm female cone and an angiosperm flower, for the main test of the analysis). This was done by dividing the lower limit of one CI (ex CI A) by the upper limit of the other (CI B) and, inversely, by dividing the upper limit of the CI A by the lower limit of CI B. We thus obtained a $\mathrm{CI}$ for the ratio $(\mathrm{CI} \mathrm{A} / \mathrm{B}=] \mathrm{min} \mathrm{A} / \mathrm{maxB}$; $\max A / \min B[)$ and then calculated the mean value of this CI; 3) testing the null hypothesis of this ratio being equal to 1 (the p-values were calculated). These analyses were performed with $R$ [14].

\section{Results}

Table 2 shows the number of unigenes of each gymnosperm that are expressed in each gymnosperm EST library, and the fraction of those for which an angiosperm ortholog could be found. The number of genes expressed in a gymnosperm organ (male or female) for which the angiosperm ortholog is detected in the angiosperms flower EST libraries is also shown.

\section{Problem with the Zamia fisheri male bank}

The number of angiosperm orthologs found among the Zamia fisheri male expressed unigenes was abnormally low, especially for Arabidopsis (10\%, i.e. approximately 4 times less than the proportion found for the female expressed genes). Most of the unigenes for which no angiosperm ortholog was found either had no similarity with any GenBank sequence or corresponded to transposable elements sequences. The number of genes with an ortholog detected in the angiosperms flower libraries was, as a consequence, also very low, which probably explains the "atypical" results found for the Z. fisheri male expressed genes comparisons.

The results of the male/female comparisons and of the main control tests are shown in table 3 .

\section{Control test}

All the reproductive/vegetative comparisons showed a significant excess of genes expressed in common in the male or female cones of a gymnosperm and in the flower of an angiosperm, compared to the genes expressed in common in the male or female cones of a gymnosperm and in the vegetative organs (leaves or pools of vegetative organs) of the angiosperms. The ratios varied from 1.2 ( $Z$. fisheri male expressed genes, but see previous section) to 4.9 (G. biloba male expressed genes) times more genes in common between the reproductive organs of the two species than between the reproductive organs of one species and the vegetative organs of the other. This excess was found for the comparisons of the three gymnosperms with the two angiosperms and using both the angiosperm flower EST libraries alone (data not shown) or a pool of angiosperm flower and inflorescence EST libraries (shown on the

Table 2 Number of sequences used, with expression and orthology information

\begin{tabular}{|c|c|c|c|c|c|c|}
\hline & & & \multicolumn{2}{|c|}{ Arabidopsis } & \multicolumn{2}{|c|}{ Oryza } \\
\hline & & & cDNA/pep & EST & cDNA/pep & EST \\
\hline & & & 38963 & 1526133 & 66710 & 1220876 \\
\hline number expressed in: & EST & unigenes & orthologs & orth exp flower & orthologs & orth exp flower \\
\hline Gb male cones & 2047 & 1481 & 892 & 326 & 884 & 645 \\
\hline Gb female cones & 2117 & 1330 & 718 & 245 & 707 & 515 \\
\hline Wm male cones & 4846 & 3325 & 2089 & 498 & 2096 & 1283 \\
\hline Wm female cones & 5283 & 3705 & 2847 & 640 & 2840 & 1792 \\
\hline Zf male cones & 4541 & 3032 & 325 & 8 & 917 & 34 \\
\hline Zf female cones & 3711 & 4021 & 1525 & 377 & 1622 & 937 \\
\hline
\end{tabular}

Gb: Ginkgo biloba; Wm: Welwitschia mirabilis; Zf: Zamia fisheri; pep: peptides; orth exp flower: orthologs expressed in the flower. 
Table 3 Ratios and p-values of the male/female and reproductive/vegetative tissues comparisons

\begin{tabular}{|c|c|c|c|c|c|}
\hline & & \multicolumn{2}{|c|}{ Arabidopsis } & \multicolumn{2}{|c|}{ Oryza } \\
\hline & & ratio & p-value & ratio & p-value \\
\hline \multirow[t]{5}{*}{ Ginkgo } & male/female & 1,089 & 0,292 & 1,01 & 1 \\
\hline & rep female/veg & 4,87 & $7,64 \mathrm{E}-38$ & 1,64 & $1,83 \mathrm{E}-26$ \\
\hline & rep male/veg & 4,912 & $4,51 E-52$ & 1,79 & $2,94 \mathrm{E}-41$ \\
\hline & rep female/leaf & 2,58 & $4,74 \mathrm{E}-21$ & 1,3 & $2,24 \mathrm{E}-10$ \\
\hline & rep male/leaf & 3,13 & $2,42 E-36$ & 1,28 & $1,11 \mathrm{E}-11$ \\
\hline \multirow[t]{5}{*}{ Welwitschia } & male/female & 1,087 & 0,17 & 0,97 & 0,186 \\
\hline & rep female/veg & 3,84 & $1,58 \mathrm{E}-70$ & 2,05 & $1,03 E-130$ \\
\hline & rep male/veg & 3,93 & $1,14 \mathrm{E}-56$ & 1,89 & $3,10 \mathrm{E}-77$ \\
\hline & rep female/leaf & 2,277 & $7,23 E-37$ & 1,32 & $4,86 \mathrm{E}-31$ \\
\hline & rep male/leaf & 2,252 & $1,23 E-28$ & 1,29 & $2,90 \mathrm{E}-18$ \\
\hline \multirow[t]{5}{*}{ Zamia } & male/female & 0,178 & $7,69 \mathrm{E}-13$ & 0,07 & $3,60 \mathrm{E}-159$ \\
\hline & rep female/veg & 4,198 & $1,79 E-45$ & 1,87 & $1,31 \mathrm{E}-52$ \\
\hline & rep male/veg & 4,898 & 0,574 & 3,93 & 0,003 \\
\hline & rep female/leaf & 2,667 & $1,78 E-28$ & 1,3 & $2,11 \mathrm{E}-13$ \\
\hline & rep male/leaf & 1,187 & 0,371 & 1,29 & 1 \\
\hline
\end{tabular}

rep: reproductive; veg: vegetative organs pool; leaf: leaves.

table), and using both the angiosperm leaves EST libraries or the angiosperm vegetative organs pool EST libraries. All the p-values are highly significant (from E10 to E-130), except the ones of the Zamia fisheri male comparisons (but see previous section).

\section{Test of the theories of the origin of the flower}

None of the gymnosperm male/female comparisons showed the excess of gymnosperm male cone genes expressed in the angiosperm flower predicted by the Mostly Male Theory for the origin of the flower. Except for the Zamia fisheri comparisons, where a significant excess of female cone expressed genes was detected among the genes expressed in the Arabidopsis or rice flower (but see first section of the Results), all the male/ female ratios were not significantly different from 1 (ratios from 0.97 to 1.09 ; p-values $>0.15$ ). The results were very similar, for each gymnosperm, when using Arabidopsis or rice as the angiosperm species.

\section{Discussion}

Our results indicate equivalent proportions of gymnosperm male and female organs genes expressed in the angiosperm hermaphrodite flower. This 1:1 ratio is not in agreement with the Mostly Male Theory prediction of an excess of male gymnosperm genes expressed in the flower of the angiosperms. We can think of different explanations for this non-detection of differences between male and female genes.
A first explanation would be that the OOM/OOF theories are correct. This would be in agreement with the expression studies by Vásquez-Lobo and collaborators [15] on gymnosperm LEAFY-like genes that aimed to further test an important observation for the MM theory: of the two LEAFY-like genes in gymnosperms, only one paralog was kept in angiosperms, and this paralog seemed to have a male-specific expression pattern [1]. These analysis do not find male vs. female-specific expression for the two LEAFY-paralogs in several gymnosperm species, and thus do not support distinctive functions of the two LEAFY-like genes in specifying male and female reproductive organs, which is compatible with the OOM/OOF theories but not with the MM one. However, it should be emphasized that while the MM theory predicts a significant difference between the proportions of the two types of expressed genes (which we were able to test and not able to find) the corresponding predictions of the OOM and OOF theories, i.e. a "significant equivalence" of the proportions of expressed genes, may not be strictly tested. In other words, our work does not falsify the OOM/OOF theories, but it is not able to verify them.

The absence of differences may also mean that expression divergence between gymnosperms and angiosperms is too great to allow these kind of comparisons. Whole genome duplications in the angiosperm lineage, in particular, could contribute to this expression divergence because of neo or subfunctionalisation of gene duplicates orthologous to male or female gymnosperm single genes. Nonetheless, our control experiment revealed that the proportion of orthologous genes expressed in the reproductive organs (both male and female) of the gymnosperms and in the angiosperms flower is significantly higher than the proportion of orthologous genes expressed in the reproductive organs of the gymnosperms and in the angiosperms vegetative tissues. This was found for all the species and libraries tested, and is not what we expect only by chance. The results of the control experiment thus seem to indicate that lack of signal in the data is not the right explanation for the $1: 1$ ratio.

One last possible explanation would be that female and male ancestral characteristics of the angiosperm flower may not be measured by the number of genes expressed in common with female and male tissues of the ancestor, but that differences between sexes are due to only a few genes or are quantitative, i.e. due to the level of expression of the (eventually the same) genes. The fact that this might be a real obstacle to the analysis is suggested by the relatively widespread capacity of stamen tissues to undergo feminization. The ectopic production of ovules, stigmatic tissues or valve-type tissues by stamens, or even their complete transformation into 
carpels (called pistillody) as is the case in some papaya genotypes [16], indicate that somatic tissues of the stamen can switch developmental programs locally and relatively late in development [17-19]. The genetic control of the process is not well understood, but work in wheat has showed that pistillody is caused in this species by alterations to the class-B MADS-box gene expression pattern in given lines following cytoplasmic substitution through recurrent backcrossing [20]. The fact that in the early diversification of the angiosperms stamen evolution has been viewed as more labile or changing more rapidly [21], further indicates that malefemale structures may share a large set of common gene networks. An alternative explanation for the male/ female shared expression patterns would concern genes expressed in the ovules. The prediction of the MM theory specifically excludes ovule-expressed gymnosperm (female) genes. It could be that enough ovule expressed genes have been retained (or redeployed) in the angiosperm flower to mask any differences among genes not derived from ovules.

Distinction between these alternative explanations would need quantitative expression data. The available EST data, comprising normalized libraries and libraries of relatively small size, do not allow us to make quantitative comparisons. Microarrays could provide such information - unfortunately such data are not available for gymnosperm reproductive structures.

Another interesting analysis would be to concentrate on the typical angiosperm flower feature, the organ for which the different theories propose a different origin the carpel. For the MM theory, the carpel tissue has a male origin (except for the ovules), while the OOM and OOF theories propose an ancestral female identity for all the female tissues in the hermaphrodite angiosperm flower. We have used affymetrix microarray data from Arabidopsis http://affymetrix.arabidopsis.info/, including expression data from the carpel, and performed the same kind of comparisons between male and female (the main test) and reproductive and vegetative (the control experiment) gymnosperm genes (EST were used for the expression data of gymnosperms) expressed in the angiosperm flower. No coherent ratios of reproductive expression over vegetative expression were obtained in the control experiment, i.e. no excess of gymnosperm reproductive tissues genes over vegetative tissues genes was found to be expressed in the angiosperm flower. This means that we cannot trust the male/female expression ratios observed. Mixing EST and microarray expression data, with different thresholds of detection, is probably preventing the detection of any eventual significant signal.

\section{Conclusions}

In conclusion, we have tested the MM theory prediction of gene expression comparison between gymnosperms and angiosperms reproductive structures using all the adequate data available up to now. Our results do not support the MM theory prediction, but further analysis, using quantitative and more detailed expression data (namely ovule and carpel angiosperm expressed genes and eventually male and female specific gymnosperm expressed genes) are needed to determine if the MM theory is, or is not, the correct explanation for the origin of the hermaphrodite flower.

\section{Acknowledgements}

We thank Gabriel Marais for stimulating discussions and very useful suggestions in the design of the project and interpretation of data, Jean Lobry and Sylvain Mousset for help with statistical analysis and Sylvain Mousset also for help with R software. We also want to thank Michael Frohlich for having proposed a testable evolutionary theory and an anonymous reviewer for his constructive comments and corrections. This project was supported by the Agence Nationale de la Recherche (grant number ANR-08-JCJC-0109).

\section{Author details}

'Université de Lyon, F-69622, Lyon, France; Université de Lyon 1, Villeurbanne; CNRS, UMR5558, Laboratoire Biométrie et Biologie Évolutive, Villeurbanne, France. ${ }^{2}$ Université de Lyon, F-69622, Lyon, France; École Normale Supérieure de Lyon, Lyon; Laboratoire de Reproduction et Développement des Plantes, Lyon, France.

\section{Authors' contributions}

RT and MC analysed and interpreted the data. RT conceived and designed the project, wrote the manuscript and supervised the work. IN and DM helped in the conception of the study, the interpretation of data and the revision of the manuscript. All the authors have given final approval of the version to be published.

Received: 15 December 2009 Accepted: 3 August 2010

Published: 3 August 2010

\section{References}

1. Frohlich MW, Chase MW: After a dozen years of progress the origin of angiosperms is still a great mystery. Nature 2007, 450(7173):1184-9.

2. Soltis DE, Bell CD, Kim S, Soltis PS: Origin and early evolution of angiosperms. Ann N Y Acad Sci 2008, 1133:3-25.

3. Pennisi E: On the Origin of Flowering Plants. Science 2009, 324(5923):28-31.

4. Theißen G, Becker A: Gymnosperm Orthologues of Class B Floral Homeotic Genes and Their Impact on Understanding Flower Origin. Critical Reviews in Plant Sciences 2004, 23(2):129-148.

5. Frohlich MW, Parker DS: The Mostly Male theory of flower evolutionary origins: from genes to fossils. Systematic Botany 2000, 25(2):155-170.

6. Theissen G, Becker A, Kirchner C, Munster T, Winter K-U, Saedler H: How land plants learned their floral $A B C s$ : the role of MADS-box genes in the evolutionary origin of flowers. In Developmental Genetics and Plant Evolution Taylor \& Francis, LondonCronk QCB, Bateman RM, Hawkins JA 2002, 173-205.

7. Frohlich MW: An evolutionary scenario for the origin of flowers. Nat Rev Genet 2003, 4(7):559-66.

8. Albert VA, Soltis DE, Carlson JE, Farmerie WG, Wall PK, llut DC, Solow TM, Mueller LA, Landherr LL, Hu Y, Buzgo M, Kim S, Yoo MJ, Frohlich MW, PerlTreves R, Schlarbaum SE, Bliss BJ, Zhang X, Tanksley SD, Oppenheimer DG, Soltis PS, Ma H, DePamphilis CW, Leebens-Mack JH: Floral gene resources 
from basal angiosperms for comparative genomics research. BMC Plant Biol 2005, 5:5.

9. Huala E, Dickerman A, Garcia-Hernandez M, Weems D, Reiser L, LaFond F, Hanley D, Kiphart D, Zhuang J, Huang W, Mueller L, Bhattacharyya D, Bhaya D, Sobral B, Beavis B, Somerville C, Rhee SY: The Arabidopsis Information Resource (TAIR): a comprehensive database and web-based information retrieval, analysis, and visualization system for a model plant. Nucleic Acids Res 2001, 29(1):102-5.

10. Ouyang S, Zhu W, Hamilton J, Lin H, Campbell M, Childs K, ThibaudNissen F, Malek RL, Lee Y, Zheng L, Orvis J, Haas B, Wortman J, Buell CR: The TIGR Rice Genome Annotation Resource: improvements and new features. NAR 2007, , 35 Database: D846-851.

11. Yuan Q, Ouyang S, Wang A, Zhu W, Maiti R, Lin H, Hamilton J, Haas B, Sultana R, Cheung F, Wortman J, Buell CR: The institute for genomic research Osa1 rice genome annotation database. Plant Physiology 2005, 138:18-26

12. Brenner ED, Katari MS, Stevenson DW, Rudd SA, Douglas AW, Moss WN, Twigg RW, Runko SJ, Stellari GM, McCombie WR, Coruzzi GM: EST analysis in Ginkgo biloba: an assessment of conserved developmental regulators and gymnosperm specific genes. BMC Genomics 2005, 6:143.

13. Wasmuth JD, Blaxter ML: prot4EST: translating expressed sequence tags from neglected genomes. BMC Bioinformatics 2004, 5(1):187.

14. R Development Core Team: R: A language and environment for statistical computing. R Foundation for Statistical Computing, Vienna, Austria 2007.

15. Vázquez-Lobo A, Carlsbecker A, Vergara-Silva F, Alvarez-Buylla ER, Piñero D, Engström P: Characterization of the expression patterns of LEAFY/ FLORICAULA and NEEDLY orthologs in female and male cones of the conifer genera Picea, Podocarpus, and Taxus: implications for current evo-devo hypotheses for gymnosperms. Evol Dev 2007, 9(5):446-59.

16. Storey WB: Pistillate Papaya Flower: A Morphological Anomaly. Science 1969, 163:401-405.

17. Bowman JL, Sakai H, Jack T, Weigel D, Mayer U, Meyerowitz EM: SUPERMAN, a regulator of floral homeotic genes in Arabidopsis. Development 1992, 114(3):599-615.

18. Gaiser JC, Robinson-Beers K, Gasser CS: The Arabidopsis SUPERMAN Gene Mediates Asymmetric Growth of the Outer Integument of Ovules. Plant Cell 1995, 7(3):333-345

19. Krizek BA, Meyerowitz EM: The Arabidopsis homeotic genes APETALA3 and PISTILLATA are sufficient to provide the $B$ class organ identity function. Development 1996, 122(1):11-22

20. Hama E, Takumi S, Ogihara Y, Murai K: Pistillody is caused by alterations to the class-B MADS-box gene expression pattern in alloplasmic wheats. Planta 2004, 218:712-720

21. Hufford $L:$ The origin and early evolution of angiosperm stamens. The Anther: Form, Function, and Phylogeny Cambridge University Press, CambridgeD'Arcy W, Keating R 1996, 58-91.

doi:10.1186/1471-2148-10-240

Cite this article as: Tavares et al:: Testing the recent theories for the origin of the hermaphrodite flower by comparison of the transcriptomes of gymnosperms and angiosperms. BMC Evolutionary Biology 2010 10:240.

\section{Submit your next manuscript to BioMed Central and take full advantage of:}

- Convenient online submission

- Thorough peer review

- No space constraints or color figure charges

- Immediate publication on acceptance

- Inclusion in PubMed, CAS, Scopus and Google Scholar

- Research which is freely available for redistribution

Submit your manuscript at www.biomedcentral.com/submit 